\title{
Non-Uniform Grid-Based Routing in Sensor Networks
}

\author{
Robert Akl and Priyanka Kadiyala \\ Department of Computer Science and Engineering \\ University of North Texas \\ Denton, Texas \\ Email:rakl@cse.unt.edu
}

\author{
Mohamad Haidar \\ Department of Electrical Engineering \\ Ecole de Technologie Superieure \\ Montreal, Quebec, Canada \\ Email: mohamad.haidar.1@ens.etsmtl.ca
}

\begin{abstract}
A non-uniform grid-based coordinated routing design in wireless sensor networks is presented. The conditions leading to network partition and analysis of energy consumption that prolongs the network lifetime are studied. We implement routing in heavily populated sensor networks. By maintaining constant values for parameters such as path loss exponent, receiver sensitivity and transmit power, and varying between uniform and non-uniform grids, we observe energy consumption patterns for each of the grid structures, and infer from the network lifetime the better suited grids for uniformly and randomly deployed sensor nodes.
\end{abstract}

Keywords-Sensor Networks; Non-uniform grids; routing protocols; energy consumption

\section{INTRODUCTION}

Wireless sensor networks, which are a form of ad hoc networks, are wireless networks consisting of autonomous sensor nodes, communicating with each other over wireless links. Each node in a sensor network consists of a central processing unit (CPU), memory, a Radio Frequency (RF) transceiver, and a power source (usually a battery).

An important aspect in wireless sensor networks is the battery lifetime of the node. Energy efficiency is a main challenge in wireless sensor networks and energy use is dominated by the energy required to keep the nodes active and running. Several energy conserving mechanisms have been proposed to extend the lifetime of the network, such as Span [1], Geographic Adaptive Fidelity (GAF) [2], Sparse Topology and Energy Management (STEM) [3], Adaptive Self-Configuring sEnsor Networks Topologies (ASCENT) [4], Clusterbased Energy Conservation (CEC) [5], and Adaptive Fidelity Energy-Conserving Algorithm (AFECA) [5]. Also, various topologies have been proposed for saving energy in sensor networks including cluster, link, grid, and diffusion, which were adapted to route packets across the sensor network. Among these topology approaches, the grid-based approach, as put forward in the GAF algorithm is more suited for sensor networks, since the grid topology can dynamically be configured with the configuration of the nodes.

In [6], the authors look at grid-based coordinated routing in wireless sensor networks. The underlying routing protocol is based on flooding [7], but unlike flooding, grid-based coordinated routing reaches only selected nodes in the field. Sensor nodes are randomly deployed over a sensor field, which is divided into square shaped grids, of user-defined sizes. One and only one node in each grid is elected as the coordinator node, which takes part in the routing process while the remaining nodes power down their radios to save energy. The source floods the network with a query message to each coordinator. When the message arrives to the sink node, the sink node sends information by tracing a route back to the source node. This process continues until a coordinator node in the route runs out of energy. Each node in the network is assigned an identification number or ID. Coordinator nodes are elected based on the ID's. The node with the highest ID in the grid is elected to be the coordinator. Once this node runs out of energy, the next highest ID node is elected as the coordinator. The process continues until the network is partitioned and the connection between the source and sink is lost. This method employs load balancing to keep the nodes running for a long time.

A directed grid topology from the source node to the sink node is proposed in [8]. This grid is built with respect to the diagonal line between the source and sink nodes. Here, the sink node can move around in the network and therefore, the topology of the grid can vary according to the positions of the source and sink nodes. What determines the distance between the grids is the average transmission cost, unlike in the previous scheme. There are two metric parameters for selecting a grid node: the distance to the location of the ideal grid node and the residual power. A cost parameter has been defined as the metric to select a grid node. The next hop is determined by the node with the smallest value for the cost parameter. There are two contributions of this proposal, namely the optimal grid distance is derived from the transmission cost point of view. Also, the routing scheme can be used for one sink and single or multiple sources.

In [9], several variants of grid-based routing are proposed for different environments. The authors mention that grid-based routing requires as few grids as possible to participate while ensuring network connectivity. The idea that keeps the network connected to one node per grid is required to stay active. This is contradicted with the argument that a largely reduced subset of grids can still preserve the same degree of coverage.

This paper reveals variants of grid-based routing schemes, which reduce the number of grids that are required to support routing while supporting network connectivity. In addition, the authors show that diagonal routing with a different side length of grids outperforms 
rectilinear routing. The above-mentioned grid-based schemes are common with the fact that they propose routing schemes for a uniform grid structure. In [10], a non-uniform grid structure is proposed for the GAF protocol, by deducing the relationship between the optimal radio range and traffic in the network. The minimum energy consumption characteristic range is not a constant but varies with the amount of traffic. Optimal range increases as the loaded traffic decreases. To save energy by radio range adjustment, the network is divided into sections of different sizes according to a derived range-traffic relationship. The number of grid sections is not a free parameter as in the case of the GAF protocol. The authors demonstrate that lower energy consumption is achieved by the non-uniform virtual grid routing, as compared to the values for the uniform grid.

The remainder of this paper is organized as follows: Section II defines the problem and the objective pursued in the paper. Section III describes our non-uniform gridbased routing protocols. Section IV provides simulations and results for various non-uniform grid-based structures, uniform grid structure, and the traditional flooding algorithm. Finally, Section V concludes the paper.

\section{PRoBlem DeFINITION}

\section{A. Problem Description and Motivation}

The major challenge posed by sensor networks is the wasteful usage of resources. It is important to keep the nodes active for as long as possible. Hence, energy usage becomes a serious issue in sensor networks. Since network partition is recognized as a major problem in densely populated sensor networks [4], the motivation of our work is to study network partition and energy consumption in sensor networks.

\section{B. Objective}

This paper studies the conditions leading to network partition and analyzes energy consumption to prolong the network lifetime. We focus on implementing routing in a densely populated sensor networks. By maintaining constant values for parameters such as path loss exponent, receiver sensitivity, transmit power, and varying between uniform and non-uniform grids, we observe energy consumption patterns for each of the grid structures and infer from the network lifetime the better suited grids for uniformly and randomly deployed sensor nodes.

\section{Contributions}

The main contributions of this paper are:

- Designing and implementing non-uniform gridbased routing with different types of nonuniform grids;

- Maintaining load balancing among the sensor nodes;

- Determining the better type of grid suitable for deployment for different node densities.

\section{NON-UNIFORM GRID BASED ROUTING}

\section{A. Uniform Grid-Based Coordinated Routing}

In uniform grid-based coordinated routing, information reaches only selected nodes in the field grids by making only one node alive for each grid, while the rest of the nodes in that grid are sleeping so as to conserve their battery life. In each grid, the coordinator participates in routing as long as the amount of energy in that coordinator is above a certain threshold value. When the energy drops below the threshold, a new coordinator is elected for that grid. The source transmits information to the sink through the active coordinators, and the sink traces a route back to the source. The process of flooding continues till the nodes participating in the routing run out of energy, when new coordinators are elected and a new route back to the source from the sink is calculated. The source starts flooding by sending a query message to all the neighbor coordinators, which flood other coordinators in the network till the message reaches the sink node. Each coordinator node in grid-based routing has three states, namely, routing, warning, and depleted states. When coordinator nodes in a particular route die, or run out of energy, new coordinators are elected to replace the old nodes.

\section{B. Non-Uniform Grid-Based Coordinated Routing}

Uniform grid-based routing is efficient when the distribution of the nodes in the sensor field is uniform. Varying the grid sizes (non-uniform) in the network extends the lifetime of the network. We consider three different non-uniform grids for simulations. Figures 1 to 3 show the different types of grid structures that are used.

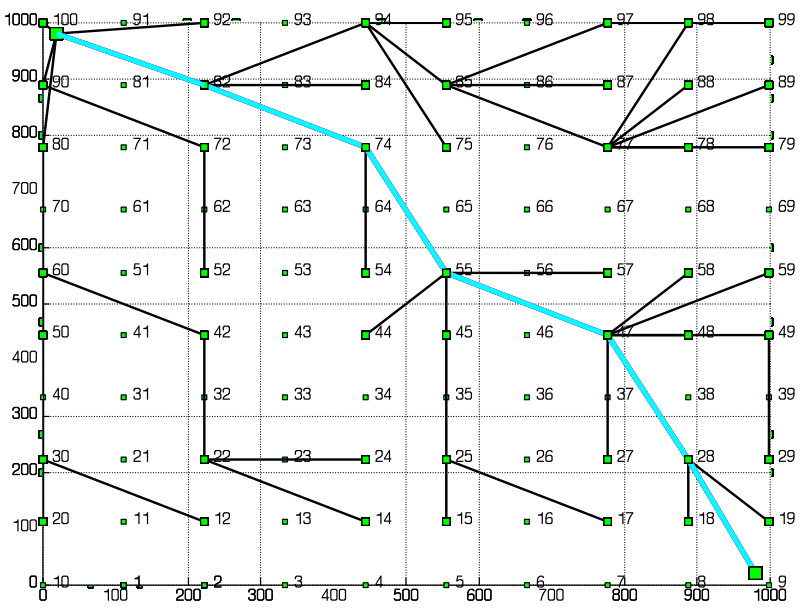

Figure 1. Topology showing flooding for 100 nodes uniformly distributed in the field for the alternating non-uniform grid structure.

In Figure 1, the alternating non-uniform grid structure is depicted where 100 sensor nodes are uniformly deployed across the study area. The alternating grid includes $100 \times 100 \mathrm{~m}^{2}$ squares (small grids) and $200 \times 200 \mathrm{~m}^{2}$ squares (large grids). 
Figure 2 shows the source non-uniform structure where 100 sensor nodes are deployed uniformly in the study area. The area containing the source node (top left) is divided into small grid squares of $100 \times 100 \mathrm{~m}^{2}$ each whereas the area containing the sink node (bottom right) is divided into grid squares $200 \times 200 \mathrm{~m}^{2}$ each.

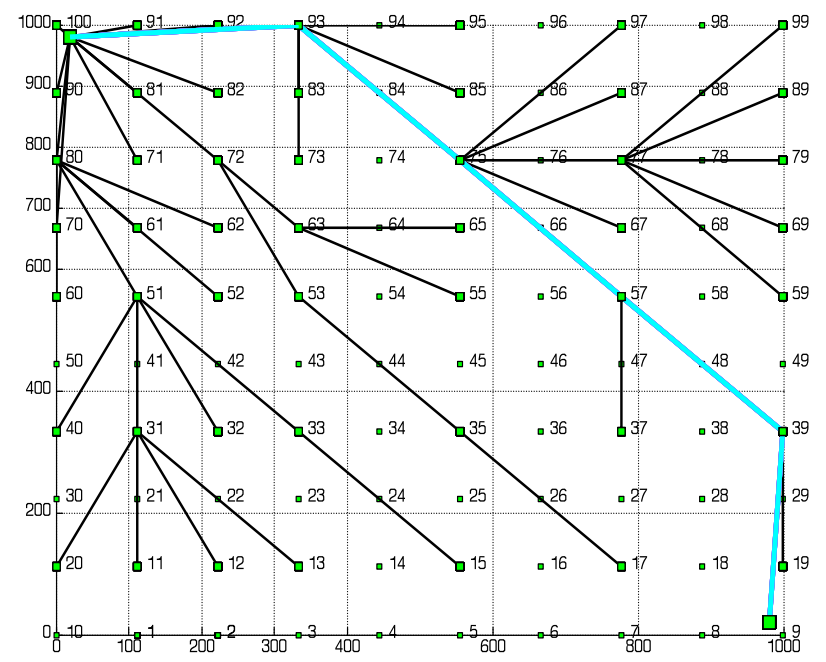

Figure 2. Simulation topology showing the source non-uniform grid structure.

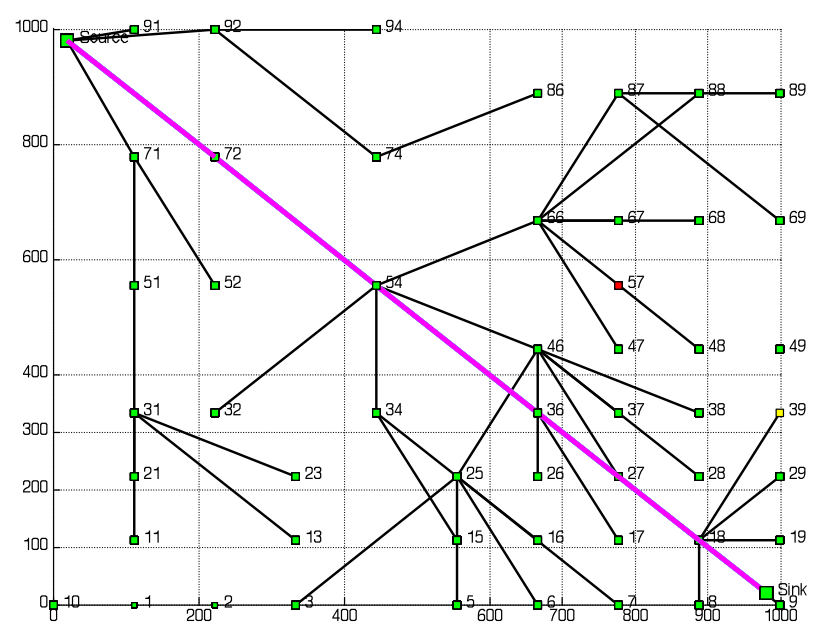

Figure 3. Simulation topology showing the sink non-uniform structure.

Finally, the sink non-uniform grid structure with 100 sensor nodes deployed uniformly across the field is presented in Figure 3. The vicinity around the source node (top left) is divided into $200 \times 200 \mathrm{~m}^{2}$ square grids while the area in the vicinity of the sink node is divided into $100 \times 100 \mathrm{~m}^{2}$ square grids.

\section{Grid Size}

Factors such as the transmission range of the transmitter (or the transmit power) and the sensitivity of the nodes affect the grid size. If either the grid size is too large or the coordinator nodes are far from each other, this will lead to early partition of the network. Thus, a link between the nodes cannot be formed even if the nodes are alive in each grid.

\section{Load-Balancing}

To utilize the nodes to their maximum lifetime, our grid-based routing protocol employs load balancing. All the nodes in the network share the coordinator node role to ensure fair usage of node resources. Each node in the network is initially assigned a rank based on the amount of energy in the node. The node with the lowest rank is elected as the coordinator. To ensure load balancing, in our algorithm the node IDs are not considered to elect coordinator nodes. Initially, since all the nodes have the same rank, one node per grid is randomly elected as the coordinator node for that grid. Once transmissions to and from the source begin, the node energy gradually depletes. If the energy of the node is greater than $25 \%$ of its battery life, the rank of that node is incremented by one, and if the energy drops to or less than $25 \%$ of battery life, the rank of that node is incremented by two, and the node has to be put to sleep. When such a node is detected in the route, the link between the source and sink is disrupted as one of the coordinator nodes in now almost dead. Hence, the source node has to re-flood the network once new coordinator nodes are elected in place of the nodes that have energies equal to or less than $25 \%$ battery life. The new coordinator nodes are the nodes that have a lower rank, more energy, and can handle routing for a longer time. Therefore, by maintaining load balancing, our gridbased routing protocol increases network lifetime. The process of node re-election continues till the network is partitioned and no link can be established between the source and the sink.

\section{Simulation AND Results}

This section presents the real-time simulations and results of the non-uniform grid-based coordinated routing protocol. Results compare the uniform grid-based coordinated routing with our three different types of nonuniform grids. Simulations were carried using MATLAB software.

\section{A. Assumptions and Parameters}

\section{1) The Energy Model}

Sensor nodes consume energy while in idle mode (listening mode). So, one of the major constraints on the wireless ad hoc sensor networks is the excessive energy consumption, which leads to diminishing the network lifetime. It is important to note that the energy spend by a node in the transmitting, receiving, and idle modes may not be the same. The idle:receive:transmit ratio of energy is shown to be 1.0: 1.05: 1.4 in [11], 1.0: 2: 2.5 in [12], and 1.0: 1.2: 1.7 in [13]. In our case, the energy spent by a node for idle listening is 1.0 unit, reception is 1.5 units, and transmission is 2.0 units. A counter array keeps track of the energy left in each node. If the node is elected as a coordinator node, it loses 1.0 unit of energy. Then, if the same node is used for transmission of data between nodes, it loses another unit of energy (i.e. a total of 2 units), but if it is a coordinator and only receives the information from a node and does not transmit the information to another coordinator, then, the node loses only 0.5 units of energy (for a total of 1.5 units). If a unit is not a coordinator but still is idle listening it uses 1.0 unit of energy every one time-unit of simulation time. 
2) Parameters Affecting Routing in the Network

The following parameters (node deployment, node density, receiver sensitivity, transmission range, and node energy) affect the lifetime of the network and are assumed to have the following values:

- Node deployment: uniform and random.

- Node density: $100,200,400$, and 1000 sensors

- Grid size: varies with type of grid being used

- Receiver Sensitivity: $-90 \mathrm{dBm}$

- Transmit power: $-2 \mathrm{dBm}$

- Path loss exponent: 3.5

- Node energy: 50 units

3) Sensor Field

The number of sensor nodes in the network is varied between 100 and 1000 nodes. The network consists of two fixed nodes that are of infinite energy, the source node (always top left) and the sink node (always bottom right). This assumption applies irrespective of the type of deployment of the nodes. Simulations are thereby observed for different node densities to analyze the scalability of the non-uniform grid-based coordinated routing protocol.

\section{B. Analysis of the Results}

For a given node density, the efficiency of the different grid structures is analyzed by comparing the network alive times along with the total transmission of each structure. Thus, three cumulative graphs are provided:

- The total transmissions for each grid structure.

- The network lifetime for each grid structure.

- The energy depletion graph.

The total transmissions allowed in the network are important to assure that the network actually allows a fair amount of information to be exchanged as long as the network is alive. Network time is kept track of by a timer that starts once the network starts to flood and stops when there is no communication link between the source and the sink nodes. Normalized energy is defined as the ratio of the total current energy of all nodes to the total energy of all nodes at the start of the simulation.

Figure 4 shows total transmissions permissible in the network for a node density of 100 nodes for all the grid structures. The source non-uniform grid structure provides the highest number of transmissions since the highest density of coordinators is near the source (where information is being generated). This allows for a lot of transmissions to be initiated before the network is fragmented

Figure 5 presents the network lifetime of the different grid structures with 100 nodes deployed randomly. The alternating non-uniform grid structure is providing better performance results with the longest network lifetime. This grid structure seems to provide the better balance between dense and scarce coordinator layout thus prolonging the network from being fragmented. This notion is confirmed when examining energy depletion over time in Figure 6.
Figure 6 shows the energy plot of different grid structures for 100 nodes resulting in a gradual decline of energy in the network with time. The maximum network lifetime is nearly 900 time units for the alternating nonuniform grid structure, and this structure better utilizes the entire energy in the network since the energy gradually decreases from 1.0 unit to nearly 0.28 units. This is due to the variable grid sizes that take into account the transmission range of transmitters and sensitivity of nodes providing a good balance for the layout of coordinators.

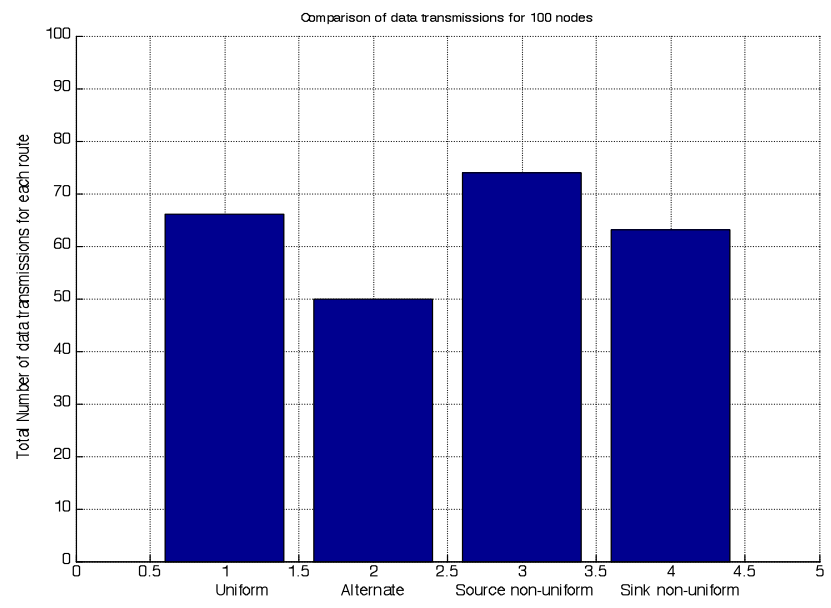

Figure 4. Total transmissions allowed for the node density of 100 nodes deployed randomly for the different grid structures.

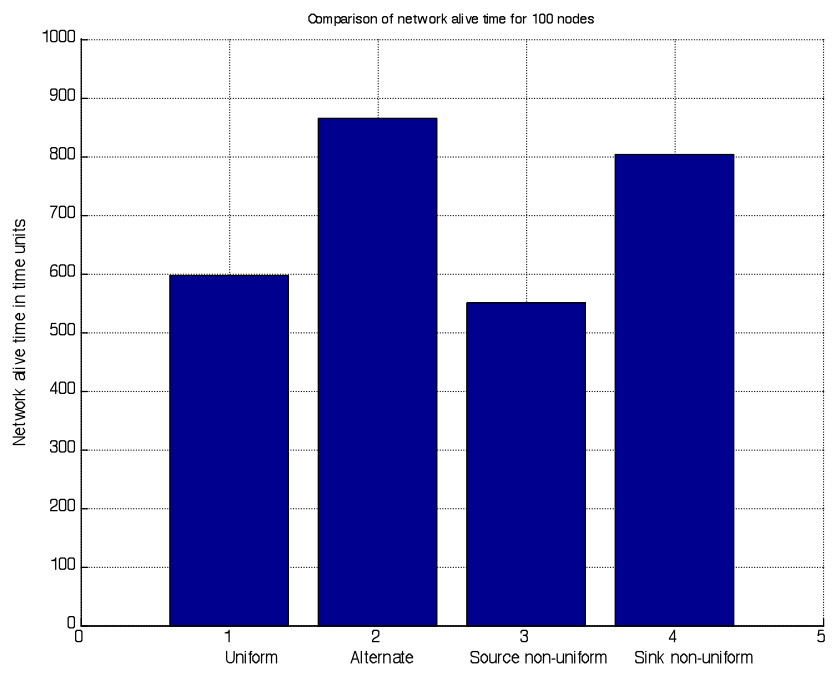

Figure 5. Network lifetime for different grid structures for 100 nodes deployed randomly across the sensor field.

\section{Summary of Results}

In varying the node density from hundreds to a thousand, we have analyzed the network lifetime for random node deployment. It has been sown that the nonuniform grid-based coordinated routing protocol is more effective than the uniform one. The comparison of network lifetime for the different grid structures with varying node density is shown in Table I. 


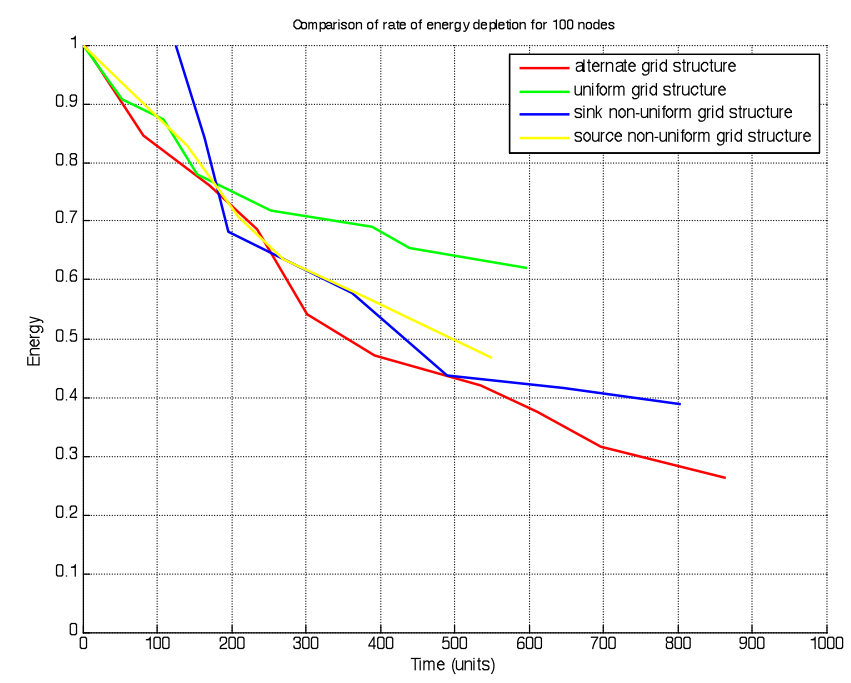

Figure 6. Normalized energy vs. simulation time performance for the different grid structures.

Table I. Comparison of network lifetime for uniform and non-uniform grid structures

\begin{tabular}{|c|c|c|c|c|}
\hline $\begin{array}{c}\text { NODE } \\
\text { DENSITY }\end{array}$ & $\begin{array}{c}\text { Lifetime for } \\
\text { Uniform } \\
\text { grid } \\
\text { (time units) }\end{array}$ & $\begin{array}{c}\text { Lifetime for } \\
\text { Alternating } \\
\text { non - } \\
\text { uniform } \\
\text { grid (time } \\
\text { units) }\end{array}$ & $\begin{array}{c}\text { Lifetime for } \\
\text { Source non- } \\
\text { uniform } \\
\text { grid } \\
\text { (time units) }\end{array}$ & $\begin{array}{c}\text { Lifetime for } \\
\text { Sink non- } \\
\text { uniform } \\
\text { grid } \\
\text { (time units) }\end{array}$ \\
\hline 100 nodes & 600 & 880 & 550 & 800 \\
\hline 200 nodes & 680 & 1250 & 780 & 1170 \\
\hline 400 nodes & 1800 & 1840 & 1660 & 1250 \\
\hline 1000 nodes & 3000 & 4500 & 4000 & 4300 \\
\hline
\end{tabular}

From Table I, it is shown that the alternating nonuniform grid structure is the better non-uniform grid structure for randomly deployed wireless sensor networks. Figure 7 shows the simulation topology for alternating non-uniform grid for 400 nodes.

\section{CONCLUSION}

The proposed non-uniform grid-based routing protocol was derived from the grid-based routing protocol. It follows the grid-based routing protocol in conserving power and surpasses uniform grid routing in dense wireless sensor networks. By using the non-uniform gridbased coordinated routing protocol the lifetime of the network was improved by a factor around 1.5 times. Future work will target the implementation on motes, mobility of nodes, and irregular distribution of nodes into dense and sparse areas.

\section{REFERENCES}

[1] B. Chen, K. Jamieson, H. Balakrishnan, and R. Morris, "Span: an Energy-efficient Coordination Algorithm for Topology Maintenance in Ad hoc Wireless Networks", Wireless Networks, Volume 8, pp. 481-494, 2002.

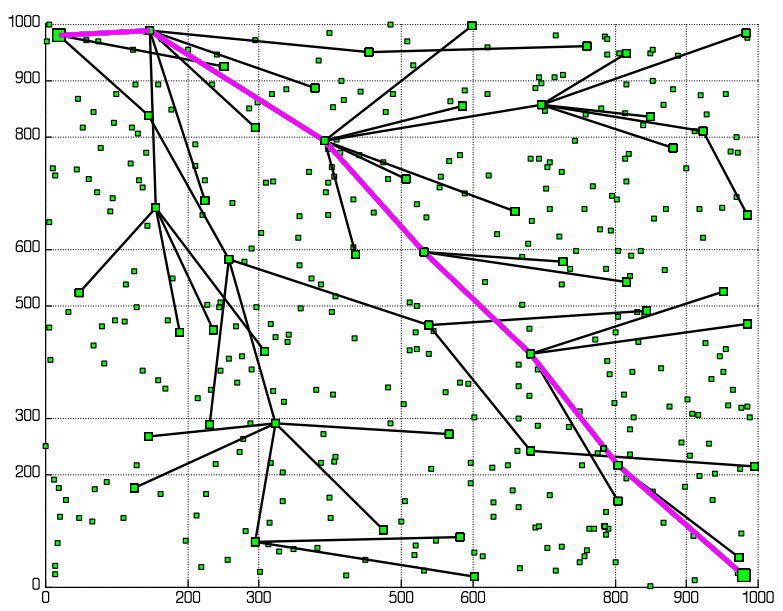

Figure 7. Simulation topology showing flooding and routing in the alternating non-uniform grid structure for 400 nodes.

[2] Y. Xu, J. Heidemann, and D. Estrin, "Geography-informed energy conservation for ad hoc routing", Proceedings of the Seventh Annual ACM/IEEE International Conference on Mobile Computing and Networking (ACM Mobicom), 2001.

[3] C. Schurgers, V. Tsiatsis, and M.B.Srivastava, "STEM: Topology management for energy efficient sensor networks", IEEE Aerospace Conference Proceedings, pp. 1099 - 1108, 2002.

[4] A.Cerpa and D. Estrin, "ASCENT: adaptive selfconfiguring sensor networks topologies", IEEE Transactions on Mobile Computing, pp. 272-285, 2004.

[5] Y. Xu, S. Bien, Y. Mori, J. Heidemann, and D. Estrin, "Topology Control Protocols to Conserve Energy in Wireless Ad hoc Networks," Center for embedded Network Sensing. Papers, 2003.

[6] R. Akl and U. Sawant, "Gid-based Coordinated Routing in Wireless sensor Networks," Consumer Communications and Networking Conference, pp. 860-864, 2007.

[7] S. Dai, X. Jing, L. Li, "Research and analysis on routing protocols for wireless sensor networks," Proceedings of the International Conference on Communications, Circuits and Systems, 2005.

[8] Y.-W. Chen and C.-S. Kuo, "Integrated design of gridbased routing in wireless sensor network," Advanced Information Networking and Applications, pp. 625-631, 2007.

[9] Z. Baoxian and H.T. Mouftah, "Efficient grid-based routing in wireless multi-hop networks," Proceedings of the $10^{\text {th }}$ IEEE Symposium on Computers and Communications, pp. 367-372, 2005.

[10] Q. Gao, K. J. Blow, D. J. Holding, I. W. Marshall, and X. Peng, "Routing analysis and energy efficiency in wireless sensor networks," IEEE $6^{\text {th }}$ CAS Symposium on Emerging Technologies: Mobile and Wireless communications, 2004.

[11] M. Stemm and R. Katz, "Measuring and Reducing Energy Consumption of Network Interfaces in Hand-held Devices," IEICE Transactions on Communications, pp. 1125-1131, 1997.

[12] O. Kasten, "Energy Consumption," $\mathrm{http://www.inf.ethz.ch/Kasten/research/bathtub/energy}$ consumption.html, 2001.

[13] B. Chen, K. Jamieson, H. Balakrishnan, and R. Morris, "Span: an Energy-efficient Coordination Algorithm for Topology Maintenance in Ad hoc Wireless Networks", Wireless Networks, Volume 8, pp. 481-494, 2002. 